\title{
Constraining scatter in the stellar mass-halo mass relation for haloes less massive than the Milky Way
}

\author{
Magdelena Allen, ${ }^{1 \star}$ Peter Behroozi ${ }^{\circledR 2}$ and Chung-Pei Ma ${ }^{1,3}$ \\ ${ }^{1}$ Department of Astronomy, University of California, Berkeley, CA 94720, USA \\ ${ }^{2}$ Department of Astronomy and Steward Observatory, University of Arizona, Tucson, AZ 85721, USA \\ ${ }^{3}$ Department of Physics, University of California, Berkeley, CA 94720, USA
}

Accepted 2019 July 5. Received 2019 July 3; in original form 2018 December 13

\begin{abstract}
Most galaxies are hosted by massive, invisible dark matter haloes, yet little is known about the scatter in the stellar mass-halo mass relation for galaxies with host halo masses $M_{\mathrm{h}} \leq 10^{11} \mathrm{M}_{\odot}$. Using mock catalogues based on dark matter simulations, we find that two observable signatures are sensitive to scatter in the stellar mass-halo mass relation even at these mass scales; i.e. conditional stellar mass functions and velocity distribution functions for neighbouring galaxies. We compute these observables for 179,373 galaxies in the Sloan Digital Sky Survey (SDSS) with stellar masses $M_{*}>10^{9} M_{\odot}$ and redshifts $0.01<z<0.307$. We then compare to mock observations generated from the Bolshoi-Planck dark matter simulation for stellar mass-halo mass scatters ranging from 0 to 0.6 dex. The observed results are consistent with simulated results for most values of scatter $(<0.6 \mathrm{dex})$, and SDSS statistics are insufficient to provide firm constraints. However, this method could provide much tighter constraints on stellar mass-halo mass scatter in the future if applied to larger data sets, especially the anticipated Dark Energy Spectroscopic Instrument Bright Galaxy Survey. Constraining the value of scatter could have important implications for galaxy formation and evolution.
\end{abstract}

Key words: galaxies: evolution-galaxies: formation-galaxies: haloes-galaxies: statistics.

\section{INTRODUCTION}

In the $\Lambda \mathrm{CDM}$ paradigm, galaxies grow at the centres of virialized, self-bound dark matter haloes. Halo formation is hierarchical, such that smaller self-bound satellite haloes can be found within the virial radii of larger haloes; haloes that are not contained within a larger virialized structure are known as central haloes.

While galaxy stellar mass correlates with halo mass, this correlation is not perfect. At halo masses $M_{h}>10^{12} \mathrm{M}_{\odot}$, there are multiple ways to estimate scatter in the galaxy-halo connection, including galaxy clustering, group catalogues, direct $\mathrm{X}$-ray masses, and satellite kinematics. These methods have converged on 0.150.23 dex of stellar mass scatter for such haloes, with no apparent dependence on halo mass (More et al. 2009; Reddick et al. 2013; Tinker et al. 2017; Kravtsov, Vikhlinin \& Meshcheryakov 2018).

Considerably less is known about the stellar mass scatter for lower mass haloes. The shape of the stellar mass-halo mass relation results in galaxy formation becoming rapidly more inefficient for haloes with masses lower than $M_{\mathrm{h}} \sim 10^{12} \mathrm{M}_{\odot}$ (Behroozi, Conroy \& Wechsler 2010; Moster et al. 2010; Behroozi, Wechsler \& Conroy 2013c; Moster, Naab \& White 2013; Garrison-Kimmel et al. 2014).

^E-mail: m.allen@berkeley.edu
Thus, low-mass galaxies have fewer satellites (limiting group catalogue and satellite kinematics approaches), no mass-dependence in their bias (limiting clustering techniques; Tinker et al. 2010), and their surrounding gas is too cold to emit detectable levels of X-rays.

At the same time, there has been increased interest in the stellar mass-halo mass scatter for $M_{\mathrm{h}}<10^{12} \mathrm{M}_{\odot}$ due to the "too big to fail' problem (Boylan-Kolchin, Bullock \& Kaplinghat 2011), wherein dark matter-only simulations overpredict the numbers of dense satellites. One way to resolve this problem is for low-mass $\left(M_{\mathrm{h}} \lesssim 3 \times 10^{9} \mathrm{M}_{\odot}\right)$ satellites to have large amounts of scatter in stellar mass at fixed halo mass, which is indeed a generic prediction from hydrodynamical simulations (Sawala et al. 2016; Munshi et al. 2017). If, on the other hand, the scatter remains tight, then several authors have proposed that warm or self-interacting dark matter models are necessary to resolve the problem (BoylanKolchin et al. 2011; Elbert et al. 2015; Garcia-Cely \& Chu 2017; Garrison-Kimmel et al. 2017).

Here, we describe a method to measure scatter in lower mass haloes that is based on forward modelling. Briefly, we use abundance matching (Nagai \& Kravtsov 2005; Conroy, Wechsler \& Kravtsov 2006; Behroozi et al. 2010; Moster et al. 2010; Reddick et al. 2013) to populate haloes with galaxies in a dark matter simulation with different amounts of scatter. In this technique, galaxies in a given observed volume are assigned by rank order 
in mass to dark matter haloes (also rank ordered by mass) in an equivalent simulated volume; these assignments are then perturbed iteratively until the desired scatter is achieved. We show that velocity distribution functions (VDFs) and conditional stellar mass functions (CSMFs) are both sensitive to the level of stellar mass-halo mass scatter. Intuitively, larger scatters allow lower mass haloes to host larger galaxies, hence reducing satellite counts in CSMFs. At the same time, smaller galaxies can be hosted by larger mass haloes, thus broadening velocity distributions.

The use of two different scatter-sensitive techniques is important, as a key uncertainty is when satellite haloes (i.e. haloes within the virial radius of a larger halo) are considered merged. Generally, higher resolution simulations track satellite haloes longer (Onions et al. 2012), and so would give higher predictions for CSMFs and VDFs at fixed scatter. The same is true if satellites are tracked after disappearance using 'orphan' techniques (e.g. Kitzbichler \& White 2008). Hence, using two different techniques allows for selfconsistently breaking this degeneracy.

We present the observational and simulated data sets in Section 2, our method for calculating CSMFs and VDFs in Section 3, results and discussion in Section 4, and conclusions in Section 5. The analysis here adopts a flat, $\Lambda \mathrm{CDM}$ cosmology $\left(\Omega_{\mathrm{M}}=0.307, \Omega_{\Lambda}=\right.$ $0.693, h=0.678, n_{s}=0.96, \sigma_{8}=0.823$ ) consistent with the Planck 2015 results (Ade et al. 2016). Stellar masses assume a Chabrier (2003) IMF, and halo masses use the virial spherical overdensity definition from Bryan \& Norman (1998).

\section{DATA}

\subsection{Observations}

Here, we describe the selection of 179,373 Sloan Digital Sky Survey (SDSS) target galaxies with $M_{*}>10^{9} \mathrm{M}_{\odot}$ and $0.01<z<0.307$ (Section 2.1.1), the corrections applied (Section 2.1.2), and the method of error calculation (Section 2.1.3).

\subsubsection{Catalogue}

Redshifts are taken from the SDSS Release 10 (Ahn et al. 2014) and have over 90 per cent completeness for galaxies brighter than the SDSS $r$-band apparent magnitude limit of 17.77. As determined in section 3 of Behroozi et al. (2015), this corresponds to a stellar mass completeness limit as a function of redshift given by:

$17.77=r<-0.25-1.9 \log _{10}\left(\frac{M_{*}}{M_{\odot}}\right)+5 \log _{10}\left(\frac{D_{L}(z)}{10 p c}\right)$,

where $M_{*}$ is the stellar mass and $D_{L}(z)$ is the luminosity distance for our assumed cosmology. Median total stellar masses and star formation rates (SFRs) are from the MPA-JHU value-added catalogue (Kauffmann et al. 2003; Brinchmann et al. 2004), updated for the imaging and spectroscopy in the SDSS Data Release 7 (Abazajian et al. 2009) and both calculated assuming Chabrier (2003) initial mass functions (IMFs). All galaxy targets are taken with $z>0.01$ to minimize the effect of peculiar velocities on their inferred distances.

As described in Section 3.1, we use isolation criteria to preferentially select central galaxies for this analysis. The fraction of isolated galaxies was found to vary significantly in regions close to survey boundaries. Hence, we excluded galaxies within any bin ( 2 degrees right ascension by 2 degrees declination) bordering survey edges (Fig. 1) from our isolated samples, but allowed such galaxies to be included in total neighbour counts. The final cut included 179,373 targets with $M_{*}>10^{9} \mathrm{M}_{\odot}$ over 5706 square degrees of sky, with a

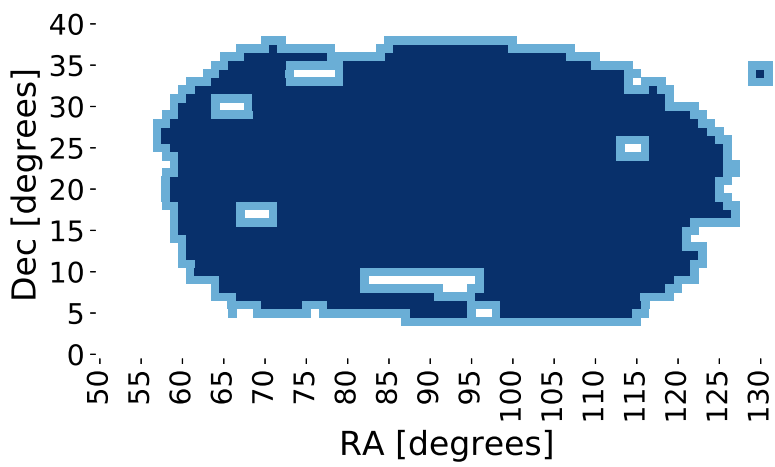

Figure 1. Map showing SDSS survey regions included (dark blue) and excluded (light blue) for this analysis.

maximum observed redshift of 0.307 and median observed redshift of 0.076 .

\subsubsection{Galaxy weights}

Close galaxy pairs (separated by less than 55 arcsec) are underselected in the SDSS due to fibre collisions, so we apply a statistical weight $w_{\mathrm{C}}$ to each galaxy in close pairs to compensate. This correction is first estimated as a function of angular separation, based on a functional model of the incompleteness of spectroscopic pairs compared to photometric pairs as defined in Patton et al. (2002), section 5.3. We take $w_{\mathrm{C}}$ to be 3.08 , as calculated for a similar SDSS sample in Patton et al. (2013), section 3.

Due to the SDSS magnitude limit given in equation (1), a galaxy of any given stellar mass is only detectable to a certain maximum redshift distance $z_{\max }$. We therefore assign each galaxy a volume correction weight

$w_{V}=1 / V\left(z_{\max }\right)$,

where $V(z)$ is the comoving volume out to redshift $z$. To correct for underselected central galaxies, we use these weights when averaging neighbour counts for the distributions detailed in Sections 3.2 and 3.3.

However, even if a central galaxy is detected at $z_{1}$, its neighbours may still be underrepresented, and so a different correction is made:

$w_{V}=\frac{1}{\max \left(V_{\mathrm{c}}, V_{n}\right)}$,

where $V_{\mathrm{c}}$ is the maximum observable volume of the central galaxy and $V_{n}$ is the same for the neighbour; $w_{\mathrm{V}}$ is thus the inverse of the maximum observable volume for the pair. The final weight applied to each galaxy is then $w_{C} w_{V}$.

\subsubsection{Error analysis}

Errors on neighbour and central galaxy counts (due to sample variance) are calculated via spatial bootstrap resampling. The observed catalogue is divided into regions of $10 \times 10$ degrees (in RA and Dec) and randomly resampled 100 times to produce reconstructed sky surveys within $<1$ per cent of the original area. Regions of $10 \times 10$ degrees are chosen to preserve local structure and resample on scales where the Universe becomes homogeneous $\left(>10 \mathrm{Mpc} \mathrm{h}^{-1}\right)$, though not much difference in error estimates is seen between using $2 \times 2$ and $10 \times 10$ degree regions in the resampling. The distributions described in Section 3 are then computed for the resampled catalogues and the standard deviation calculated. Our 
simulated catalogues are much larger in volume than our observed sample, and so we assume that the error budget is dominated by observational sample variance.

\subsection{Simulations}

Here, we describe the generation of simulated dark matter haloes (Section 2.2.1), the addition of orphan satellites (Section 2.2.2), and the process of assigning galaxy masses to account for variable scatter in the halo mass-stellar mass relation (Section 2.2.3).

\subsubsection{Dark matter simulation}

We base our mock catalogues (one for each tested value of scatter) on the Bolshoi-Planck dark matter simulation (Klypin et al. 2016; Rodríguez-Puebla et al. 2016). The simulation followed $2048^{3}$ particles ( $~ 8$ billion) each of mass $1.55 \times 10^{8} \mathrm{M}_{\odot} \mathrm{h}^{-1}$ in a periodic box of comoving side length $250 \mathrm{Mpch}^{-1}$ from $z=100$ to $z=0$, using the ART code (Kravtsov, Klypin \& Khokhlov 1997; Kravtsov \& Klypin 1999). The adopted flat $\Lambda$ CDM cosmology was consistent with Planck 2015 results $\left(\Omega_{\mathrm{M}}=0.307, \Omega_{\Lambda}=0.693\right.$, $\left.h=0.678, n_{s}=0.96, \sigma_{8}=0.823\right)$. Haloes were found using the ROCKSTAR phase-space halo finder (Behroozi, Wechsler \& Wu 2013a) and the CONSISTENT TREES merger tree code (Behroozi et al. 2013b).

\subsubsection{Orphan satellites}

A significant uncertainty with simulations is how long satellite haloes persist before disruption. In large cosmological simulations, it is often necessary to include 'orphan' satellites to match galaxy clustering (Kitzbichler \& White 2008; Behroozi et al. 2018; Moster, Naab \& White 2018); here, we generate catalogues with orphans as they are required to best match observations.

Orphan satellite haloes were added to the Bolshoi-Planck halo catalogues following the prescription in Behroozi et al. (2018). Briefly, satellites that disappear in the simulation are presumed to continue orbiting their last host halo; we integrate their continued motion using a softened gravity law:

$\dot{\mathbf{v}}=\frac{G M_{\text {host }}(<r)}{\left(r+0.1 R_{\text {vir,host }}\right)^{2}}$,

where $M_{\text {host }}(<r)$ is the total mass (including dark matter and baryons) enclosed within the satellite distance $r, R_{\text {virhost }}$ is the virial radius of the host halo, and the softening is performed to avoid unphysical hard scattering between the satellite and the host halo. The choice to include orphan haloes is motivated by comparison with observations in Appendix B.

Note that the effects of dynamical friction are not accounted for in this model, because those effects are most significant when the two interacting objects are of similar mass. Here, the vast majority of orphan satellites that disrupt in the simulation are of much smaller mass than their host haloes, and therefore the effects of dynamical friction are minimal. However, it is important to note that these effects could share parameter space with orphan lifetimes, and we do not have any good constraints on these effects.

Satellite mass-loss follows Jiang \& van den Bosch (2016), with the modification that satellites do not lose mass on infalling orbits and lose mass at twice the rate on outgoing orbits (Behroozi et al. 2018). Satellites are assumed to disrupt (merge with central halo) once their maximum circular velocity $\left(v_{\max } \equiv\right.$ $\left.\max _{R} \sqrt{G M(<R) / R}\right)$ falls below $0.6 v_{\text {Mpeak }}$, where $v_{\text {Mpeak }}$ is the maximum circular velocity at the time the halo reached peak mass. Identical merging criteria are applied to orphan and ordinary satellites: the stellar mass of the satellite is merged into the central galaxy if it is within $\sim 0.25 R_{\text {virhost }}$ and added to the diffuse halo otherwise. This prescription results in $\sim 25$ per cent more satellites independent of mass, and was found in Behroozi et al. (2018) to give the best match to galaxy autocorrelation functions.

\subsubsection{Assigning galaxy masses}

Galaxy masses are then assigned using an abundance matching approach (e.g. Marinoni \& Hudson 2002; Nagai \& Kravtsov 2005; Conroy et al. 2006; Reddick et al. 2013) to the dark matter halo catalogue (including orphans) at $z=0$. We compute galaxy number densities from the selected SDSS regions (included in Fig. 1, totalling 5706 square degrees) using the inverse volume weights described in equation (2). We compute the resulting number of galaxies as a function of mass that would be expected within the simulation volume. These galaxies are ordered by decreasing mass and assigned to haloes in order of decreasing $v_{\text {Mpeak }}$ with zero scatter. We then introduce $v_{\text {Mpeak }}$-dependent log-normal scatter of $\sigma\left(v_{\text {Mpeak }}\right)$ via the following iterative algorithm:

(i) Each scattered mass $M_{*}^{\prime}$ is drawn from a log-normal distribution centred on the assigned stellar mass $M_{*}$ and of width $\sigma\left(v_{\text {Mpeak }}\right)$. This introduces the correct scatter, but deforms the shape of the total stellar mass function.

(ii) Redo abundance matching (with the original SDSS galaxy number densities) to haloes ordered by decreasing $M_{*}^{\prime}$. This corrects the shape of the stellar mass function, but alters the distribution of the scatter.

(iii) Recompute the median $M_{*}\left(v_{\text {Mpeak }}\right)$ relation from the stellar masses assigned in step (ii). End algorithm if median relation changes by less than 1 percent from previous iteration. This step approximates the change in the median $M_{*}\left(v_{\text {Mpeak }}\right)$ relation necessary to reproduce the correct stellar mass function after adding scatter.

(iv) Assign stellar masses to haloes with zero scatter according to the computed median $M_{*}\left(v_{\text {Mpeak }}\right)$ relation and go to step (i).

This can be recognized as a simple deconvolution algorithm, as discussed further in Behroozi et al. (2010).

Because constraints on the scatter for massive haloes $\left(M_{\mathrm{h}}>\right.$ $10^{12} \mathrm{M}_{\odot}$ ) are tighter than for less massive haloes, at $\sim 0.2 \mathrm{dex}$ (Reddick et al. 2013), we adopt the following functional form for $\sigma\left(v_{\text {Mpeak }}\right)$, with a given scatter $X$ for low-mass haloes:

$$
\frac{\sigma\left(v_{\text {Mpeak }}, X\right)}{\operatorname{dex}}= \begin{cases}0.2 & \text { if } v_{\text {Mpeak }}>200 \mathrm{~km} \mathrm{~s}^{-1} \\ X & \text { if } v_{\text {Mpeak }}<120 \mathrm{~km} \mathrm{~s}^{-1} \\ X+(0.2-X) L\left(v_{\text {Mpeak }}\right) & \text { otherwise, }\end{cases}
$$

where $L\left(v_{\text {Mpeak }}\right)$ linearly interpolates from 0 to 1 as $v_{\text {Mpeak }}$ goes from 120 to $200 \mathrm{~km} \mathrm{~s}^{-1}$ :

$L\left(v_{\text {Mpeak }}\right)=\frac{v_{\text {Mpeak }}-120 \mathrm{~km} \mathrm{~s}^{-1}}{200 \mathrm{~km} \mathrm{~s}^{-1}-120 \mathrm{~km} \mathrm{~s}^{-1}}$.

This forms a bridge between the well-constrained scatter of highmass haloes and the values tested for the unknown scatter of lowmass haloes. The exact bounds on $v_{\text {Mpeak }}$ are an arbitrary modelling choice, and smoothly vary the scatter from 0.2 for $\sim 10^{12} \mathrm{M}_{\odot}$ haloes to $X$ for $\sim 10^{11} \mathrm{M}_{\odot}$ and smaller haloes. Here, we test seven different 


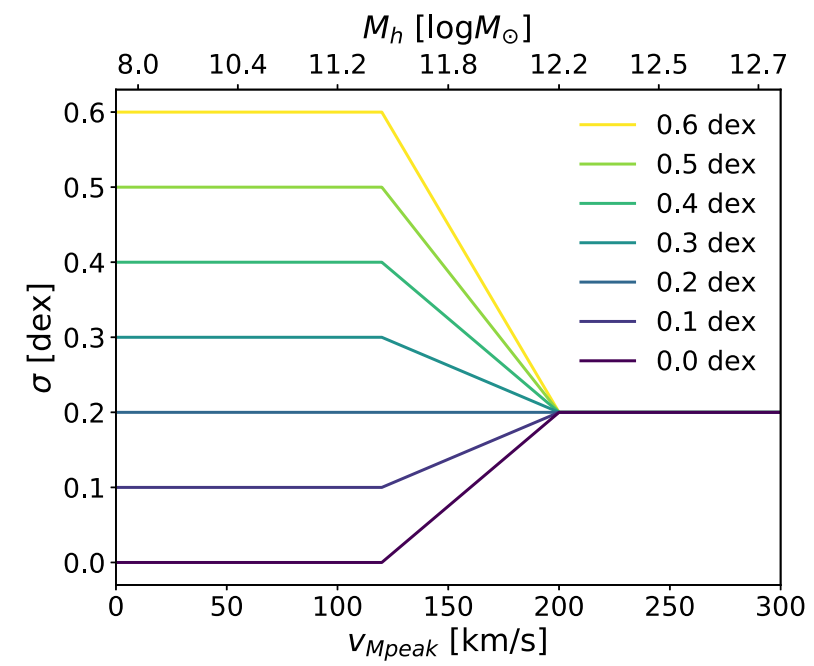

Figure 2. Functional form of scatter in dex for each tested model, as a function of the maximum circular velocity at the time the halo reached peak mass $\left(v_{\text {Mpeak }}\right)$. The equivalent peak halo mass appears on the top axis. All models are consistent with prior observable constraints; i.e. the scatter in stellar mass is $\sim 0.2$ dex for haloes of mass $M_{\mathrm{h}}>10^{12} \mathrm{M}_{\odot}$.

values of $X$, i.e. $0.0,0.1,0.2,0.3,0.4,0.5$, and 0.6 ; the resulting functions for $\sigma\left(v_{\text {Mpeak }}\right)$ are plotted in Fig. 2.

\section{METHODS}

Here, we describe methods for selecting isolated galaxies (Section 3.1) as well as for measuring CSMFs (Section 3.2) and VDFs (Section 3.3) of neighbouring galaxies around our selected galaxy samples. The same methods are applied both to the observations from the SDSS (Section 2.1) and to seven mock catalogues generated from dark matter simulations (Section 2.2). The mock catalogues each include a different stellar mass-halo mass scatter from 0 to $0.6 \mathrm{dex}$ for haloes $M_{\mathrm{h}}<10^{11} \mathrm{M}_{\odot}$; to match observed constraints on scatter for higher mass haloes, the input scatter is fixed at 0.2 dex for $M_{\mathrm{h}} \gtrsim 10^{12} \mathrm{M}_{\odot}$ and smoothly varied for $10^{11} \mathrm{M}_{\odot}<M_{\mathrm{h}}$ $<10^{12} \mathrm{M}_{\odot}$ haloes (see Fig. 2 and Section 2.2). CSMFs and VDFs are calculated separately for low-mass galaxies $\left(10^{9}-10^{10} \mathrm{M}_{\odot}\right)$ and high-mass galaxies $\left(10^{10}-10^{11} \mathrm{M}_{\odot}\right)$.

\subsection{Isolation cuts}

To constrain the scatter in the relationship between halo mass and stellar mass for central galaxies, we first apply an isolation cut to galaxies in both simulations and observations to preferentially select central galaxies. We define purity as the fraction of selected galaxies that are true central galaxies; we define completeness as the fraction of true central galaxies that pass our cut. As discussed in Appendix A, we take cuts to maximize completeness while retaining above 90 percent purity for the two galaxy mass ranges analysed (Table 1). For the lower mass sample $\left(10^{9}-10^{10} \mathrm{M}_{\odot}\right)$, we select galaxies that are the most massive within $0.5 \mathrm{Mpch}^{-1}$ projected comoving distance $r_{\mathrm{p}}$ and a redshift distance $\Delta v$ of $\pm 500 \mathrm{~km} \mathrm{~s}^{-1}\left(5 \mathrm{Mpch}^{-1}\right.$ in comoving line of sight distance). For the higher mass sample $\left(10^{10}-10^{11} \mathrm{M}_{\odot}\right)$, we select galaxies that are the most massive within $1 \mathrm{Mpch}^{-1}$ projected comoving distance and a redshift distance of $\pm 1000 \mathrm{~km} \mathrm{~s}^{-1}\left(10 \mathrm{Mpc} \mathrm{h}^{-1}\right.$ in comoving line of sight distance). Using comoving distance instead of physical distance ensures a fairer comparison between the observations (at a range of redshifts; see Section 2.1) and simulated catalogues (at a single redshift, $z=0$; see Section 2.2.3).

In the simulated galaxy catalogues, we use the distant observer approximation, so that projected distances are calculated along the $X$ and $Y$ axes, and redshift distances are calculated as $Z+\frac{v_{Z}}{H(z)}$, where $v_{Z}$ is the $Z$-velocity and $H(z)$ is the Hubble expansion rate.

In the observations, we calculate the projected comoving distance as:

$r_{\mathrm{p}}=\theta_{\mathrm{sep}} D\left(z_{2}\right)$,

where $\theta_{\text {sep }}$ is the angular distance between the two galaxies, and $D\left(z_{2}\right)$ is the comoving line-of-sight distance calculated from the redshift of the neighbouring galaxy. We use $z_{2}$ instead of the more common average of $z_{2}$ and $z_{1}$ (the redshift of the central galaxy) to minimize the difference between the volume searched around observed and simulated central galaxies due to the distant observer approximation.

\subsection{Conditional stellar mass function}

Here, we define the CSMF as the number counts of neighbouring galaxies as a function of stellar mass within a cylindrical aperture and $\pm 1.5 \mathrm{Mpch}^{-1}$ redshift distance $\left(|\Delta v|<150 \mathrm{~km} \mathrm{~s}^{-1}\right)$, averaged across all central galaxies. Neighbours are required to be galaxies within the same mass range as the sample (low-mass or high-mass) being considered. The observed CSMFs are calculated with the corrections for fibre collisions and maximum enclosed volume as described in Section 2.1.2.

For both low- and high-mass galaxies, we count neighbours within a projected distance cut of their central galaxy to maximize statistics and minimize any disagreements between the simulations and observations. This projected distance $r_{\mathrm{p}}$ is comoving, as defined in equation (7).

Average neighbour counts as a function of projected distance for low-mass and high-mass central galaxies (Fig. 3) show consistency of observed to simulated catalogues, and reveal that satellite galaxies are concentrated at small radii. As expected, the low-mass galaxies are much more sensitive to the adopted scatter model. Counts are seen to be increasing with projected distance $\gtrsim 250 \mathrm{kpch}^{-1}$ in the low-mass sample, and this is likely an artefact of the isolation cut: selecting low-mass centrals necessarily means selecting galaxies in environments that are underdense within the chosen projected distance cut. (This phenomena would not apply to high-mass centrals, and this trend is not observed for the high-mass sample.) As motivated in Appendix B2, we take neighbours $50<r_{\mathrm{p}}<$ $200 \mathrm{kpch}^{-1}$ for low-mass galaxies and neighbours $100<r_{\mathrm{p}}<$ $300 \mathrm{kpch}^{-1}$ for high-mass galaxies for the highest consistency between simulated and observed catalogues, and for the best statistics.

High values of scatter allow larger haloes to host smaller galaxies and smaller haloes to host larger galaxies, so the CSMFs of lowmass galaxies are expected to increase and the CSMFs of highmass galaxies are expected to decrease. Nonetheless, because the magnitude of the scatter for high-mass haloes is fixed, the effect on the CSMF will be much larger for low-mass than for high-mass galaxies. Low-mass galaxies clearly produce the expected trend (Fig. 4). High-mass galaxies show less distinction between scatters (Fig. 5), which is expected, but the 0.6 dex CSMF clearly deviates from the expected trend. This indicates the threshold at which scatter becomes high enough to contaminate our sample of selected central galaxies (i.e. to reduce purity). If falsely identified centrals are removed from the high-mass simulated distributions, overall counts 
Table 1. Purity and completeness of central galaxies for the chosen galaxy isolation cuts, as measured in our simulated galaxy catalogues.

\begin{tabular}{lccccc}
\hline Scatter $($ dex $)$ & Mass cut $\left(\log M_{\odot}\right)$ & $r_{\mathrm{p}} \operatorname{cut}\left(\mathrm{Mpch}^{-1}\right)$ & $\Delta v \mathrm{cut}_{\left(\mathrm{Mpc} \mathrm{h}^{-1}\right)}$ & Purity & Completeness \\
\hline 0.0 & $9-10$ & 0.5 & 5 & 0.987 & 0.533 \\
0.6 & $9-10$ & 0.5 & 5 & 0.953 & 0.513 \\
0.0 & $10-11$ & 1 & 10 & 0.982 & 0.433 \\
0.6 & $10-11$ & 1 & 10 & 0.912 & 0.407 \\
\hline
\end{tabular}

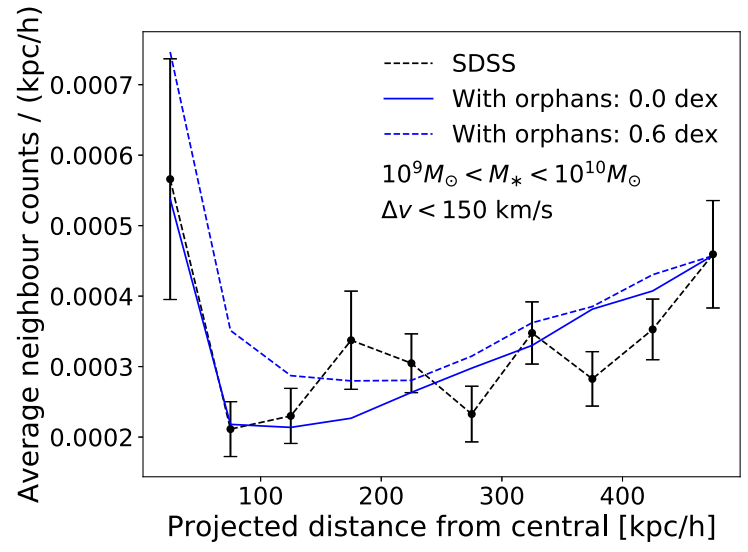

(a) Low-mass galaxies $\left(10^{9}-10^{10} \mathrm{M}_{\odot}\right)$.

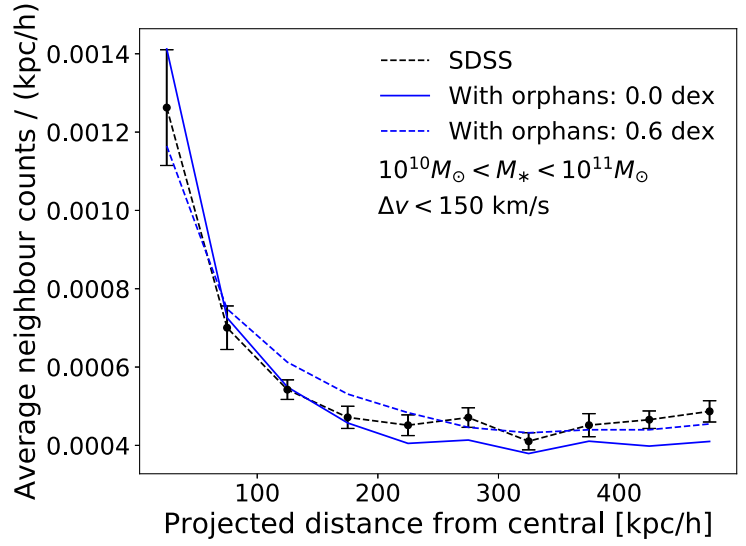

(b) High-mass galaxies $\left(10^{10}-10^{11} \mathrm{M}_{\odot}\right)$.

Figure 3. Average neighbour counts as a function of projected distance from low-mass and high-mass galaxies. Neighbours are galaxies in the same mass range within $\pm 1.5 \mathrm{Mpc} \mathrm{h}^{-1}$ in redshift distance $\left(|\Delta v|<150 \mathrm{~km} \mathrm{~s}^{-1}\right)$. The black line shows the observed distribution and the blue lines show the simulated distributions for different input scatters between stellar mass and halo mass.

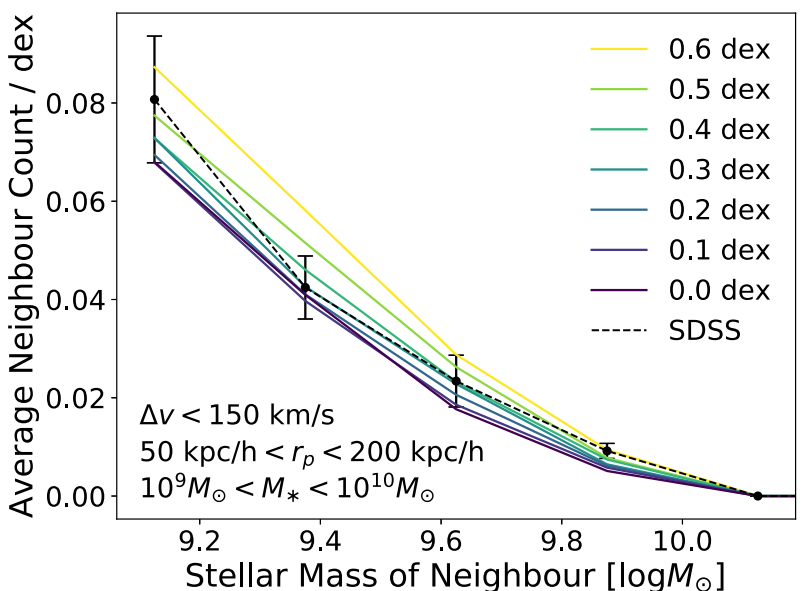

Figure 4. Comparison of observed and simulated conditional stellar mass functions for low-mass galaxies. Neighbours are galaxies in the same mass range and within $50-200 \mathrm{kpch}^{-1}$ projected and $\pm 1.5 \mathrm{Mpch}^{-1}$ redshift distance $\left(|\Delta v|<150 \mathrm{~km} \mathrm{~s}^{-1}\right)$.

are reduced and the sample shows decreasing neighbour counts with increasing scatter, as predicted (Fig. 6).

\subsection{Velocity distribution}

The VDF gives the average number of neighbours as a function of redshift distance within a circular aperture. Again, we require neighbours to belong to the same mass range as the sample of central galaxies considered and take projected distance cuts of 50 $200 \mathrm{kpch}^{-1}$ for low-mass galaxies and $100-300 \mathrm{kpch}^{-1}$ for high-

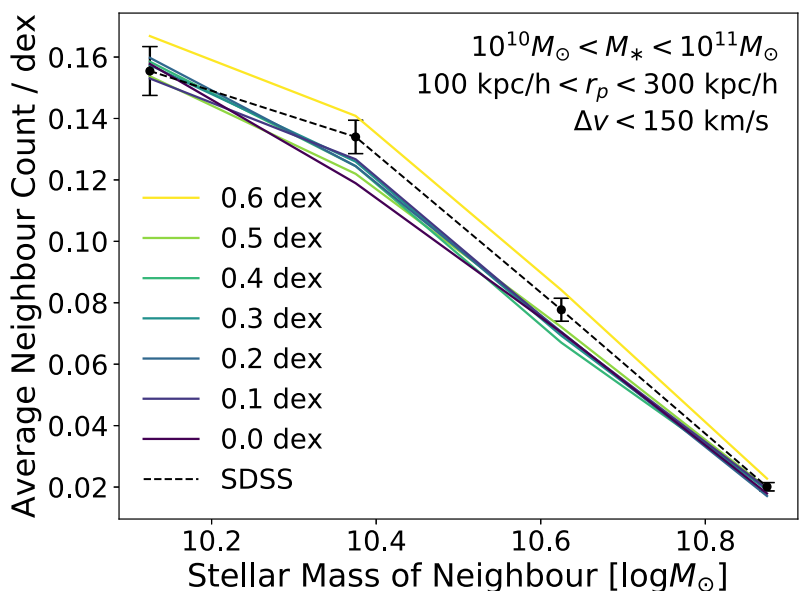

Figure 5. Comparison of observed and simulated conditional stellar mass functions for high-mass galaxies. Neighbours are galaxies in the same mass range and within $100-300 \mathrm{kpch}^{-1}$ projected and $\pm 1.5 \mathrm{Mpch}^{-1}$ redshift distance $\left(|\Delta v|<150 \mathrm{~km} \mathrm{~s}^{-1}\right)$.

mass galaxies. Observed VDFs are calculated with the corrections described in Section 2.1.2.

Higher values of scatter allow larger haloes to host smaller galaxies and vice versa, and so, like the CSMFs, VDFs are expected to increase for low-mass central galaxies and decrease for high-mass central galaxies. We find the expected trend clearly differentiated for low-mass central galaxies (Fig. 7). Again, there is little distinction in high-mass central galaxies, except for the outlying 0.6 dex VDF that does not conform to the expected trend (Fig. 8). This is the same effect seen in the CSMFs (Section 3.2), where increased scatter 


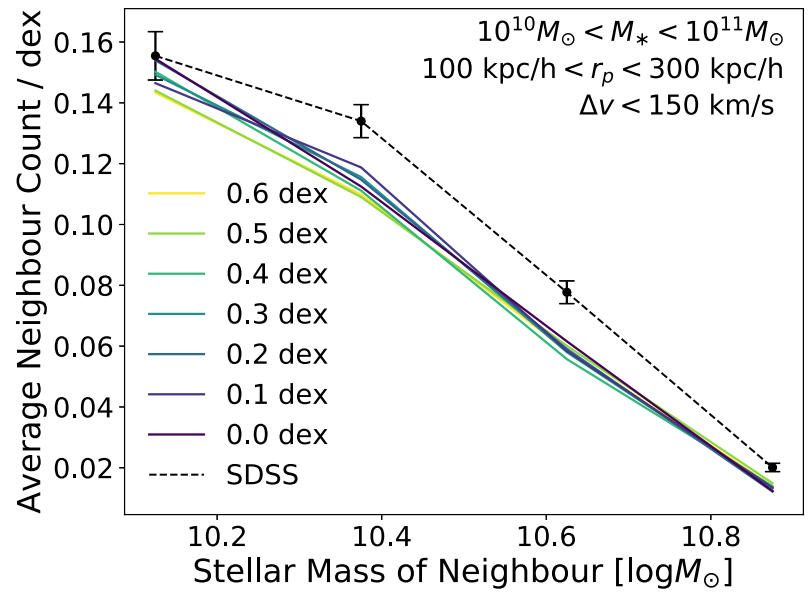

Figure 6. Comparison of observed and simulated conditional stellar mass functions for high-mass galaxies, with falsely identified centrals removed from the simulated distributions. Neighbours are galaxies in the same mass range and within $100-300 \mathrm{kpch}^{-1}$ projected and $\pm 1.5 \mathrm{Mpch}^{-1}$ redshift distance $\left(|\Delta v|<150 \mathrm{~km} \mathrm{~s}^{-1}\right)$.

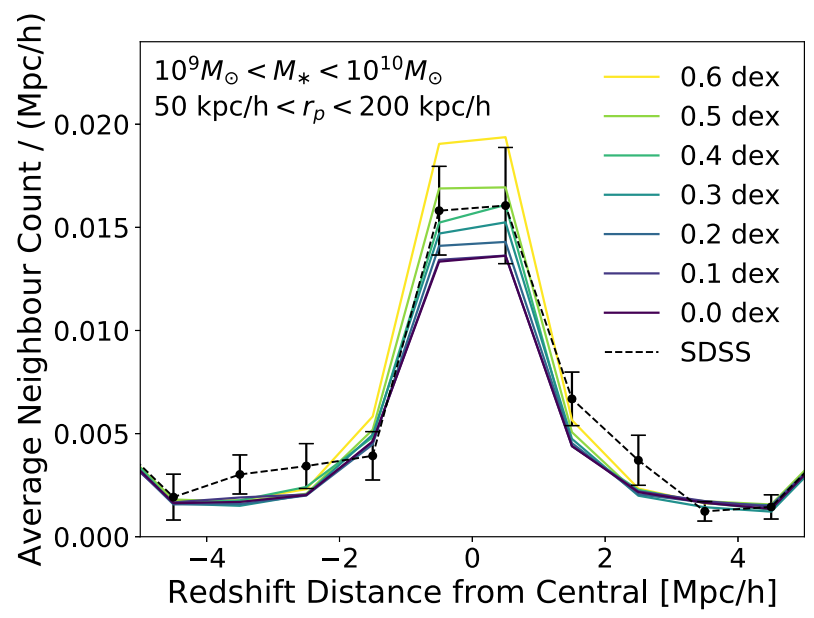

Figure 7. Comparison of observed and simulated velocity distribution functions for low-mass galaxies. Neighbours are galaxies in the same mass range and within $50-200 \mathrm{kpch}^{-1}$ projected distance. The upturn at large redshift distances is expected due to the isolation criterion (no larger galaxy within a redshift distance of $5 \mathrm{Mpch}^{-1}$ ).

causes contamination in the sample, reducing purity. With falsely identified centrals removed from the high-mass sample, the counts fall with increasing scatter, as expected (Fig. 9).

Given that our simulated catalogues have no difference for the scatter for high-mass $\left(\gtrsim 10^{12} \mathrm{M}_{\odot}\right)$ haloes, we expect (and find) that the greatest sensitivity to the input scatter comes from the CSMFs and VDFs for low-mass galaxies. Because the CSMFs and VDFs for high-mass galaxies are relatively insensitive to the input scatter, they instead serve as an important check that our adopted orphan model is realistic - i.e. that our satellite lifetimes are accurate.

\section{RESULTS AND DISCUSSION}

We compare the observed distributions to the simulations for varying values of scatter. No significant discrepancies exist between

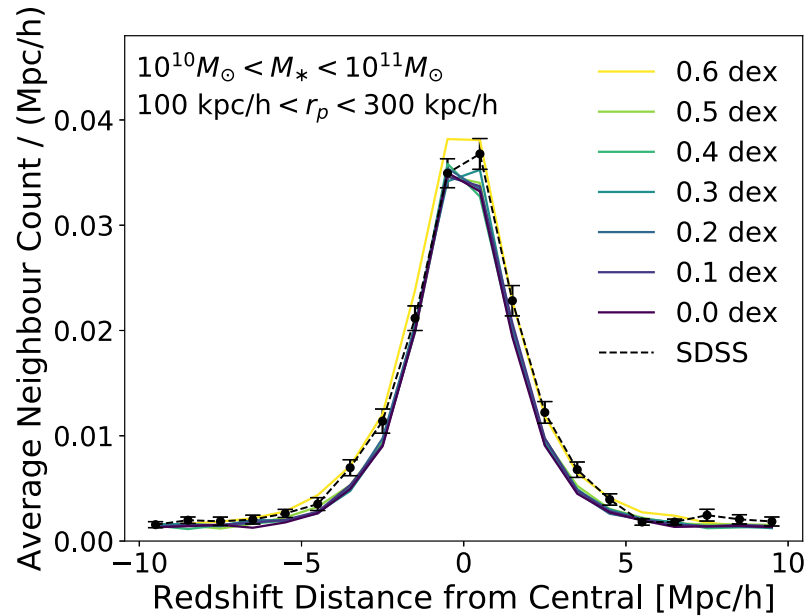

Figure 8. Comparison of observed and simulated velocity distribution functions for high-mass galaxies. Neighbours are galaxies in the same mass range and within $100-300 \mathrm{kpch}^{-1}$ projected distance.

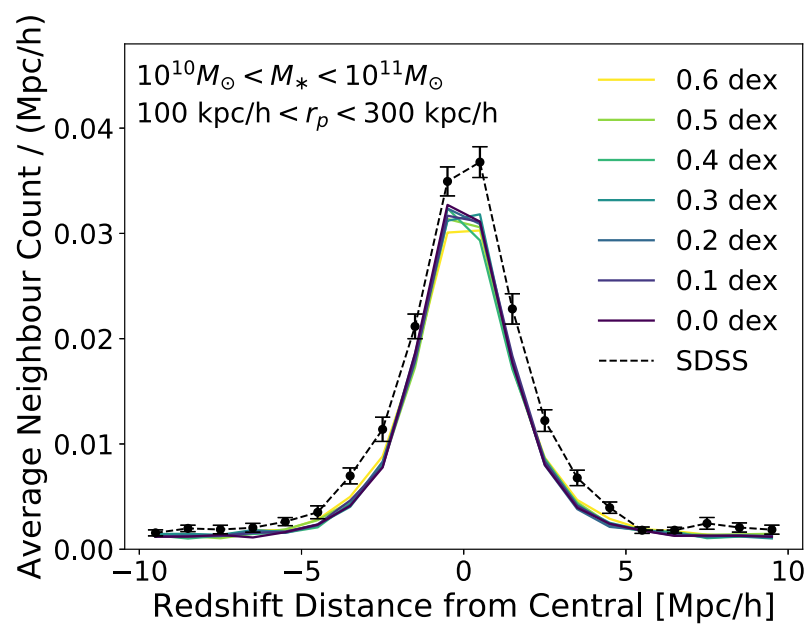

Figure 9. Comparison of observed and simulated velocity distribution functions for high-mass galaxies, with falsely identified centrals removed from the simulated distributions. Neighbours are galaxies in the same mass range and within $100-300 \mathrm{kpch}^{-1}$ projected distance.

the models and the observations (Figs 4, 7, 5, and 8), giving confidence that the models are sufficiently flexible to describe the observations.

The CSMF and VDF for low-mass central galaxies are shown in Figs 4 and 7, respectively. They indicate that the observations are most consistent with values of scatter $<0.6$ dex. The high-mass CSMF and VDF (Figs 5 and 8) show less distinction across scatters, but are consistent with the simulated catalogues, suggesting that our orphan model is correctly capturing satellite lifetimes. Thus, we cannot put tight constraints on scatter without improved statistics beyond those available in the SDSS.

The methods we developed in Section 3 nonetheless have the potential power to distinguish between different scatters for lowmass haloes and produce distributions that are consistent with the observed Universe. This method could be applied in the future to larger data sets; in particular, the upcoming Dark Energy Spectroscopic Instrument (DESI) Bright Galaxy Survey is anticipated to provide redshifts for over 10 million galaxies (DESI Collaboration 2016) compared to the $\sim 200,000$ SDSS targets in our cut, and 
the pipeline will be able to distinguish close neighbour luminosity profiles with a higher degree of precision (Schlegel et al. 2015). Even regardless of the ability to probe satellites at closer projected distances, the resulting error bars would be better by at least a factor of $\sqrt{50}$, enabling very sensitive tests for both the orphan model adopted and for the scatter in the stellar mass-halo mass relation for galaxies with $M_{\mathrm{h}}<10^{11} \mathrm{M}_{\odot}$. Based on the scatter-dependence of the low-mass CSMF (Fig. 4), such reduced error bars could constrain scatter to approximately \pm 0.05 dex and allow sensitive testing of satellite lifetimes and the orphan model described in Section 2.2.

\section{CONCLUSIONS}

We have developed a method (Section 3 ) to constrain scatter in the stellar mass-halo mass relation for central galaxies with host halo masses $M_{\mathrm{h}} \leq 10^{11} \mathrm{M}_{\odot}$ by comparing the mass and velocity distributions of neighbouring galaxies in dark matter simulations and observations. We find that the simulations produce very consistent results with the SDSS for most values of scatter $(<0.6 \mathrm{dex})$, and that SDSS statistics are inadequate to firmly constrain the scatter (Section 4). The method is nonetheless easily applied to future surveys, including the Dark Energy Spectroscopic Instrument experiment (DESI; DESI Collaboration 2016), which will conclusively measure the stellar mass-halo mass scatter for low-mass haloes.

\section{ACKNOWLEDGEMENTS}

PB was partially supported through program number HST-HF251353.001-A, provided by NASA through a Hubble Fellowship grant from the Space Telescope Science Institute, which is operated by the Association of Universities for Research in Astronomy, Incorporated, under NASA contract NAS5-26555.

\section{REFERENCES}

Abazajian K. N. et al., 2009, ApJS, 182, 543

Ade P. A. et al., 2016, Astron. Astrophys., 594, A13

Ahn C. P. et al., 2014, ApJS, 211, 17

Behroozi P., Wechsler R., Hearin A., Conroy C., 2019, MNRAS, 488, 3143

Behroozi P. S., Conroy C., Wechsler R. H., 2010, ApJ, 717, 379

Behroozi P. S., Wechsler R. H., Wu H.-Y., 2013a, ApJ, 762, 109

Behroozi P. S., Wechsler R. H., Wu H.-Y., Busha M. T., Klypin A. A., Primack J. R., 2013b, ApJ, 763, 18

Behroozi P. S., Wechsler R. H., Conroy C., 2013c, ApJ, 770, 57

Behroozi P. S. et al., 2015, MNRAS, 450, 1546

Boylan-Kolchin M., Bullock J. S., Kaplinghat M., 2011, MNRAS, 415, L40

Brinchmann J., Charlot S., White S. D. M., Tremonti C., Kauffmann G.,

Heckman T., Brinkmann J., 2004, MNRAS, 351, 1151

Bryan G. L., Norman M. L., 1998, ApJ, 495, 80

Chabrier G., 2003, PASP, 115, 763

Conroy C., Wechsler R. H., Kravtsov A. V., 2006, ApJ, 647, 201

DESI Collaboration, 2016, preprint (arXiv:1611.00036)
Elbert O. D., Bullock J. S., Garrison-Kimmel S., Rocha M., Oñorbe J., Peter A. H. G., 2015, MNRAS, 453, 29

Garcia-Cely C., Chu X., 2017, preprint (arXiv:1705.06221)

Garrison-Kimmel S., Boylan-Kolchin M., Bullock J. S., Lee K., 2014, MNRAS, 438, 2578

Garrison-Kimmel S., Bullock J. S., Boylan-Kolchin M., Bardwell E., 2017, MNRAS, 464, 3108

Jiang F., van den Bosch F. C., 2016, MNRAS, 458, 2848

Kauffmann G. et al., 2003, MNRAS, 346, 1055

Kitzbichler M. G., White S. D. M., 2008, MNRAS, 391, 1489

Klypin A., Yepes G., Gottlöber S., Prada F., Heß S., 2016, MNRAS, 457, 4340

Kravtsov A. V., Klypin A. A., 1999, ApJ, 520, 437

Kravtsov A. V., Klypin A. A., Khokhlov A. M., 1997, ApJS, 111, 73

Kravtsov A. V., Vikhlinin A. A., Meshcheryakov A. V., 2018, Astron. Lett., 44,8

Marinoni C., Hudson M. J., 2002, ApJ, 569, 101

More S., van den Bosch F. C., Cacciato M., Mo H. J., Yang X., Li R., 2009, MNRAS, 392, 801

Moster B. P., Somerville R. S., Maulbetsch C., van den Bosch F. C., Macciò A. V., Naab T., Oser L., 2010, ApJ, 710, 903

Moster B. P., Naab T., White S. D. M., 2013, MNRAS, 428, 3121

Moster B. P., Naab T., White S. D. M., 2018, MNRAS, 477, 1822

Munshi F., Brooks A. M., Applebaum E., Weisz D. R., Governato F., Quinn T. R., 2017, preprint (arXiv:1705.06286)

Nagai D., Kravtsov A. V., 2005, ApJ, 618, 557

Onions J. et al., 2012, MNRAS, 423, 1200

Patton D. R. et al., 2002, ApJ, 565, 208

Patton D. R., Torrey P., Ellison S. L., Mendel J. T., Scudder J. M., 2013, MNRAS, 433, L59

Reddick R. M., Wechsler R. H., Tinker J. L., Behroozi P. S., 2013, ApJ, 771, 30

Rodríguez-Puebla A., Behroozi P., Primack J., Klypin A., Lee C., Hellinger D., 2016, MNRAS, 462, 893

Sawala T. et al., 2016, MNRAS, 456, 85

Schlegel D. J. et al., 2015, in American Astronomical Society Meeting Abstracts \#225. Vol. 47, San Diego, California, 336.07

Tinker J. L., Robertson B. E., Kravtsov A. V., Klypin A., Warren M. S., Yepes G., Gottlöber S., 2010, ApJ, 724, 878

Tinker J. L. et al., 2017, ApJ, 839, 121

\section{APPENDIX A: ISOLATION CUTS}

Isolation cuts are applied to galaxies in both simulations at observations to select central galaxies. We choose cuts for low-mass $\left(10^{9}-10^{10} \mathrm{M}_{\odot}\right)$ and high-mass $\left(10^{10}-10^{11} \mathrm{M}_{\odot}\right)$ galaxies to maximize purity and completeness, i.e. the ratio of simulated centrals matching our centrality cut to total centrality cut or total simulated centrals, respectively. We took cuts to maximize completeness while retaining above 90 percent purity for all values of scatter; both decrease monotonically with increasing scatter, but vary by less than 10 per cent over $0.0-0.6$ dex from typical values of $\sim 95$ percent purity and $\sim 50$ per cent completeness (Table 1 in Section 3). As expected, purity rises and completeness falls with harsher isolation cuts for both low-mass and high-mass galaxies (Figs A1 and A2). 


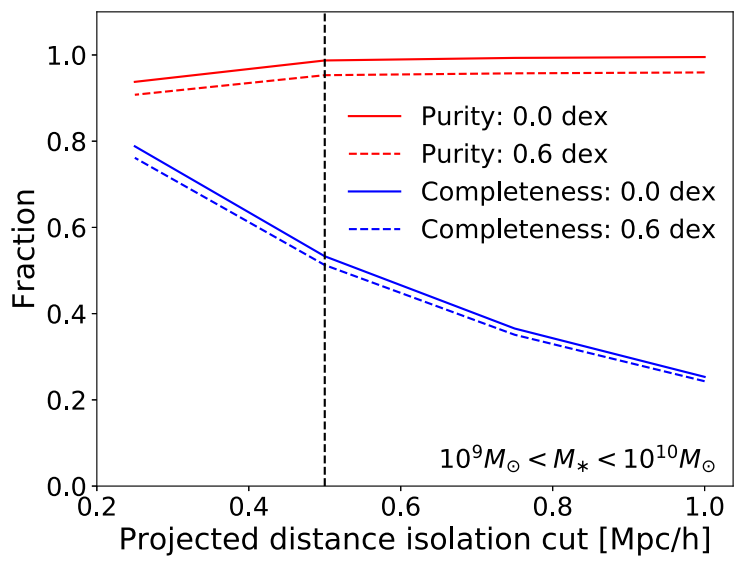

(a) Low-mass galaxies $\left(10^{9}-10^{10} \mathrm{M}_{\odot}\right)$.

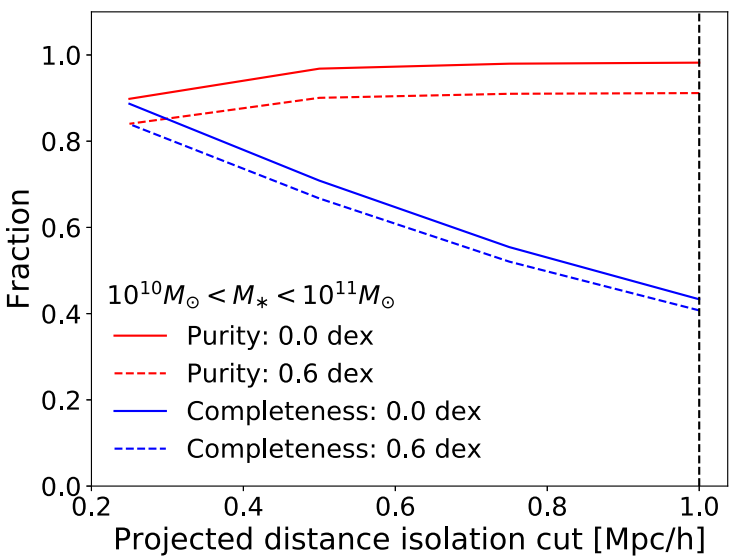

(b) High-mass galaxies $\left(10^{10}-10^{11} \mathrm{M}_{\odot}\right)$.

Figure A1. Purity and completeness for the central-satellite selection criteria for low-mass (left-hand panel) and high-mass (right-hand panel) simulated galaxies as a function of projected distance cut. Purity shown in red and completeness in blue (solid for 0.0 dex scatter, dashed for 0.6 dex scatter); the dashed vertical line indicates our chosen centrality cut.

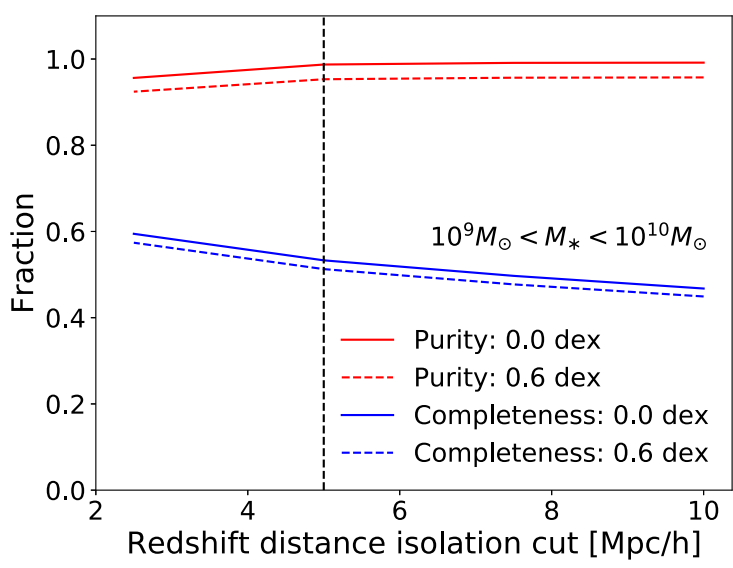

(a) Low-mass galaxies $\left(10^{9}-10^{10} \mathrm{M}_{\odot}\right)$.

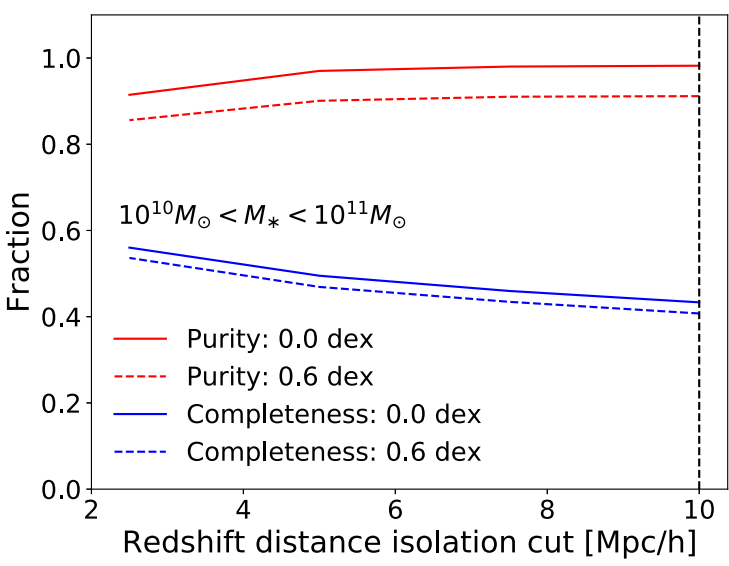

(b) High-mass galaxies $\left(10^{10}-10^{11} \mathrm{M}_{\odot}\right)$.

Figure A2. Purity and completeness for the central-satellite selection criteria for low-mass (left-hand panel) and high-mass (right-hand panel) simulated galaxies as a function of redshift distance cut. Purity shown in red and completeness in blue (solid for 0.0 dex scatter, dashed for 0.6 dex scatter); the dashed vertical line indicates our chosen centrality cut.

\section{APPENDIX B: ORPHAN HALOES IN SIMULATIONS}

Orphan satellite haloes are often included in simulations to match galaxy clustering, as described in Section 2.2. After generating catalogues with and without orphans, we find that models including orphan haloes provide a better match to observations.

\section{B1 Inclusion of orphan haloes}

Average neighbour counts as a function of projected distance from their central galaxy show agreement with the catalogue including orphans, for both low- and high-mass galaxies (Fig. B1). Errors on observations are calculated with the bootstrap resampling method described in Section 2.1.3. Clearly, catalogues generated without orphans grossly under-represent neighbours within $150 \mathrm{kpch}^{-1}$, while catalogues with orphans place these neighbour counts within a sigma for high and low scatters.

\section{B2 Motivating projected distance cuts}

The characteristics of a galaxy's nearest larger neighbour provide valuable information about clustering and serves as a measure of consistency between simulated and observed catalogues, motivating projected distance cuts on neighbours in the CSMFs and VDFs. The analysis below is carried out for catalogues adopting zero scatter, and the results are similar for $0.6 \mathrm{dex}$. The mass distributions of nearest larger neighbours show close agreement between observations and both catalogues, for low- and high-mass galaxies (Figs B2 and B3).

Notable differences are seen in the distribution of projected distance to nearest larger neighbour for very near $\left(<100 \mathrm{kpch}^{-1}\right)$ neighbours, in the case of both low- and high-mass galaxies (Figs B4 and B5). Misleadingly, the high-mass distribution appears to more closely match catalogues without orphans.

This apparent deficit in near neighbours, particularly for highmass galaxies, is likely due to observational incompleteness, i.e. overlapping luminosity profiles resulting in near neighbours going undetected. This effect would be more prominent in high-mass 


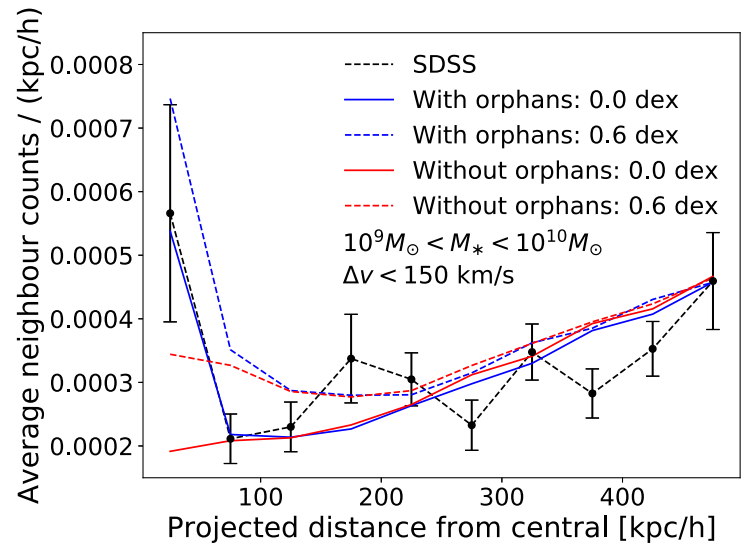

(a) Low-mass galaxies $\left(10^{9}-10^{10} \mathrm{M}_{\odot}\right)$.

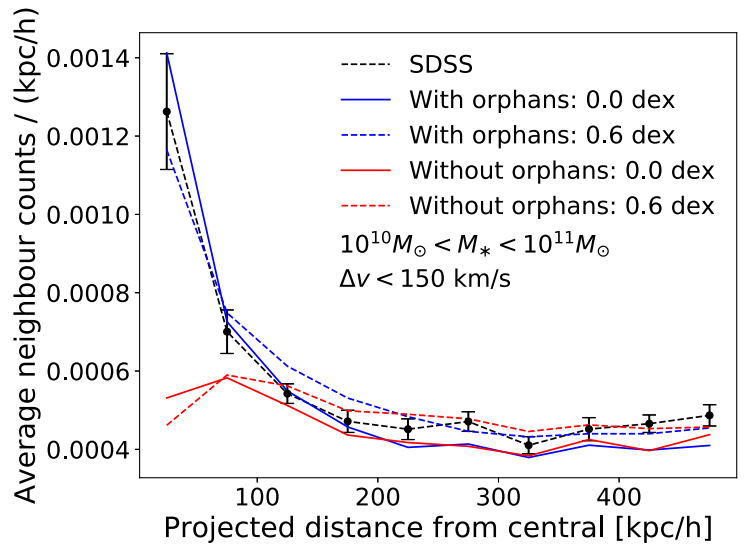

(b) High-mass galaxies $\left(10^{10}-10^{11} \mathrm{M}_{\odot}\right)$.

Figure B1. Average neighbour counts as a function of projected distance to low-mass (left-hand panel) and high-mass (right-hand panel) central galaxies, for catalogues generated with and without orphans. Neighbours are within $\pm 1.5 \mathrm{Mpch}^{-1}$ redshift distance. The black line shows the observed distribution, compared to the simulation with and without orphan haloes (blue and red lines, respectively) for different scatters.

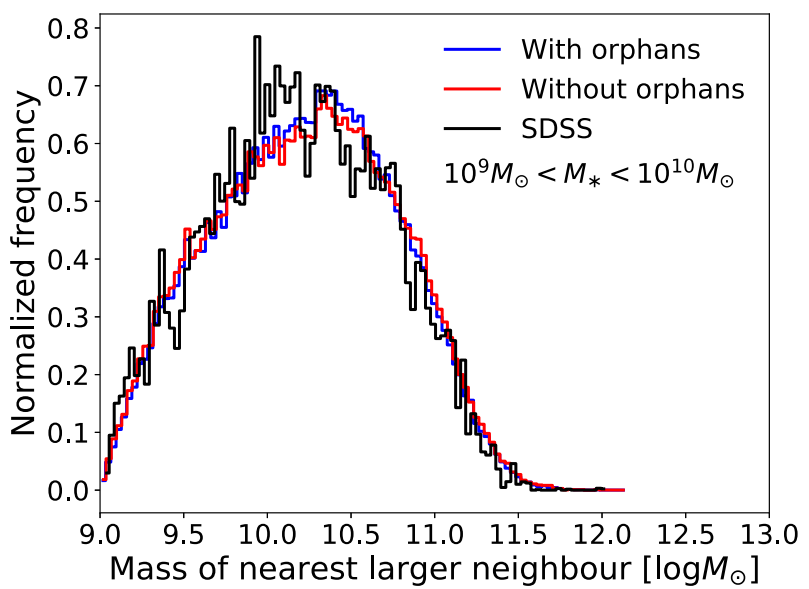

Figure B2. Normalized histogram of mass of nearest larger neighbour for high-mass galaxies (neighbours within $0.5 \mathrm{Mpch}^{-1}$ projected and $\pm 5 \mathrm{Mpch}^{-1}$ redshift distance). Compares observations (black), catalogue with orphans for (blue), and catalogue without orphans (red). Simulations are both for the model with zero scatter.

galaxies, since they have brighter luminosities. Inaccuracy in fibre collision corrections (described in Section 2.1.2) would also more greatly effect high-mass galaxies, which have higher degrees of spatial clustering. Galaxies this close may also be in the process of merging, which would further complicate disentangling them in the observational pipelines.

The distribution of velocity differences to nearest larger neighbours is shown in Figs B6 and B7; a normal distribution of noise centred on $\pm 30 \mathrm{~km} \mathrm{~s}^{-1}$ is added to the simulated velocity distributions to match uncertainties in redshift measurements. Observations are similar to both catalogues, but show slight differences from the catalogue with orphans at very close $\left(<50 \mathrm{~km} \mathrm{~s}^{-1}\right)$ redshift distances. Neighbours with small line-of-sight velocity differences may correspond to small projected distances as well, and then suffer the same issues of completeness as described above.

Overall, the differences in the distributions of nearest larger neighbour characteristics between observations and simulated catalogues (with or without orphan haloes) are small, and we use

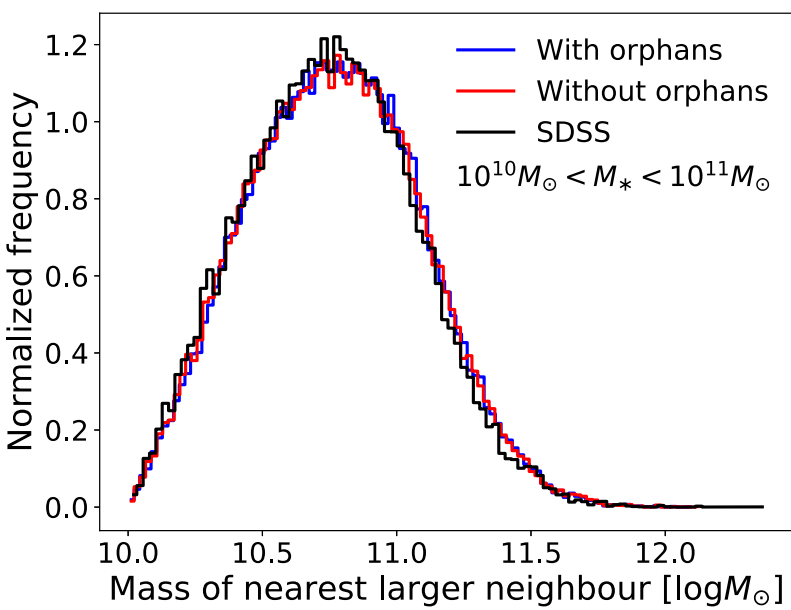

Figure B3. Normalized histogram of mass of nearest larger neighbour for high-mass galaxies (neighbours within $1 \mathrm{Mpch}^{-1}$ projected and $\pm 10 \mathrm{Mpc} \mathrm{h}^{-1}$ redshift distance). Compares observations (black), catalogue with orphans for (blue), and catalogue without orphans (red). Simulations are both for the model with zero scatter.

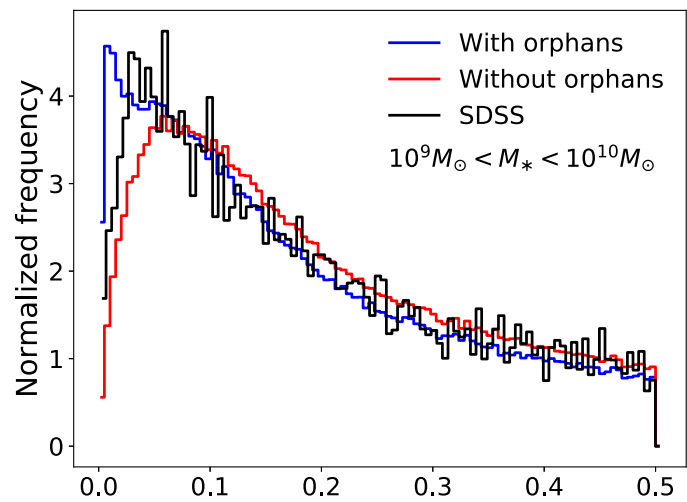

Projected distance to nearest larger neighbour [Mpc/h]

Figure B4. Normalized histogram of projected distance to nearest larger neighbour for high-mass galaxies (neighbours within $0.5 \mathrm{Mpch}^{-1} \mathrm{pro}^{-}$ jected and $\pm 5 \mathrm{Mpc} \mathrm{h}^{-1}$ redshift distance). Compares observations (black), catalogue with orphans for (blue), and catalogue without orphans (red). Simulations are both for the model with zero scatter. 


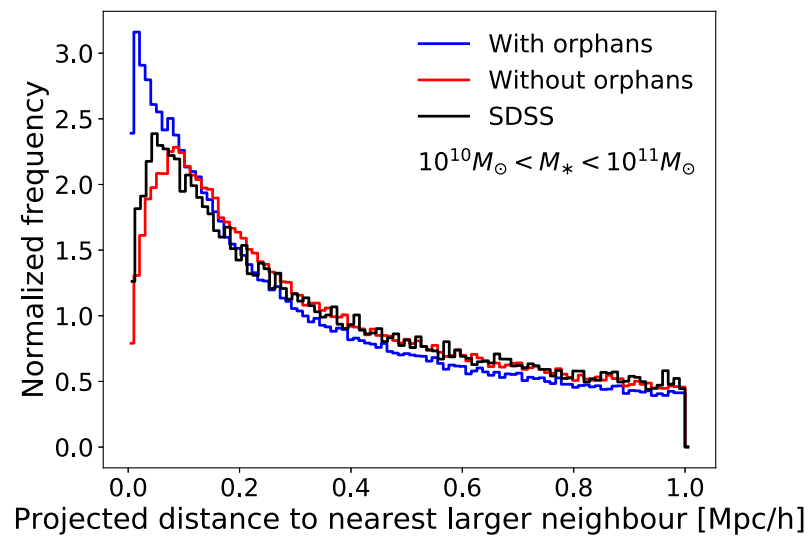

Figure B5. Normalized histogram of projected distance to nearest larger neighbour for high-mass galaxies (neighbours within $1 \mathrm{Mpch}^{-1}$ projected and $\pm 10 \mathrm{Mpc} \mathrm{h}^{-1}$ redshift distance). Compares observations (black), catalogue with orphans for (blue), and catalogue without orphans (red). Simulations are both for the model with zero scatter.

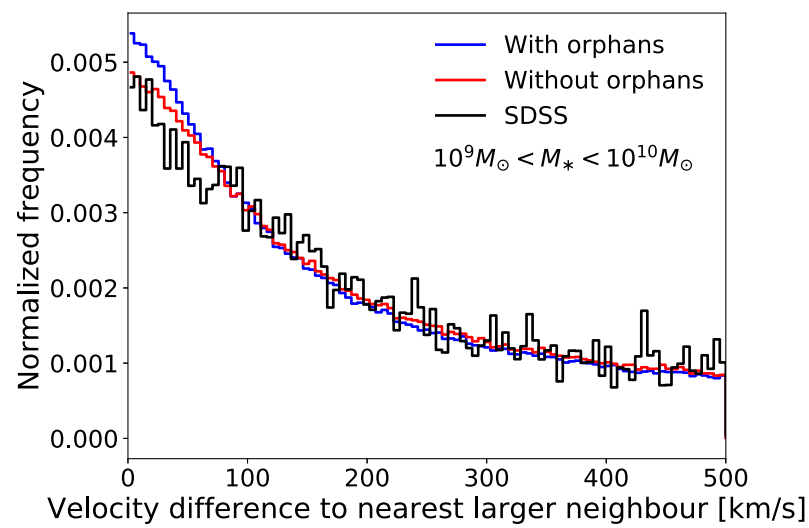

Figure B6. Normalized histogram of magnitude of velocity difference to nearest larger neighbour for high-mass galaxies (neighbours within $0.5 \mathrm{Mpch}^{-1}$ projected and $\pm 5 \mathrm{Mpch}^{-1}$ redshift distance). Compares observations (black), catalogue with orphans for (blue), and catalogue without orphans (red). Simulations are both for the model with zero scatter.

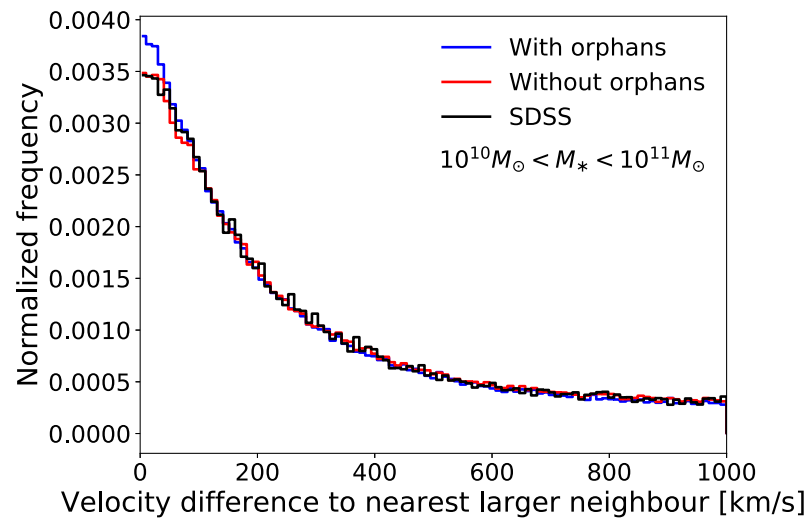

Figure B7. Normalized histogram of magnitude of velocity difference to nearest larger neighbour for high-mass galaxies (neighbours within $1 \mathrm{Mpch}^{-1}$ projected and $\pm 10 \mathrm{Mpch}^{-1}$ redshift distance). Compares observations (black), catalogue with orphans for (blue), and catalogue without orphans (red). Simulations are both for the model with zero scatter. these results to motivate a projected distance cut of $>50 \mathrm{kpch}^{-1}$ and $>100 \mathrm{kpch}^{-1}$ for the low- and high-mass CSMFs and VDFs described in Sections 3.2 and 3.3, respectively, and thus minimize these effects in our analysis.

This paper has been typeset from a $\mathrm{T}_{\mathrm{E}} \mathrm{X} / \mathrm{L} \mathrm{T} \mathrm{E} \mathrm{X}$ file prepared by the author. 